\title{
Germline editing: time for discussion
}

\author{
Discussion and regulation of genetic alterations in human germ cells and embryos is urgently needed.
}

$\mathbf{O}$ n March 5, the MIT Technology Review published an article, "Engineering the Perfect Baby," in which unnamed sources asserted that scientists in China had used clustered regularly interspersed short palindromic repeat (CRISPR)-Cas9 technology to genetically alter an early stage in vitro-fertilized human embryo, and that their results might soon be published. Germline modification of human embryos for use in reproduction is already prohibited by many countries. But the ease, speed and comparative specificity of CRISPRCas9 for genetic engineering, and its potential for germline alteration of heritable disease traits-as well as non-disease traits-have heightened the need for an immediate and global discussion of the ethical and regulatory implications of such research.

CRISPR-Cas9, transcription activator-like effector nucleases (TALENs) and zinc finger nucleases (ZFNs) have all been used to genetically engineer human somatic cells and lab animals, including monkeys, but germline alterations in human germ cells and embryos have not-at the time of writing-been reported. Moreover, off-target effects and protein engineering hurdles have made TALENs and ZFNs less-appealing candidates for use in germline editing. The CRISPR-Cas9 system has eliminated many of these hurdles.

In a Comment in Nature responding to the potential new research, Edward Lanphier of Sangamo BioSciences and colleagues call for a moratorium on all gene editing of human reproductive cells, citing ethical and safety concerns. In particular, the authors express fears that gene editing technology could be used for non-therapeutic uses and that a regulatory backlash could limit gene editing of (non-germline) somatic cells (http://www.nature.com/news/don-t-edit-the-humangerm-line-1.17111).

In contrast, other scientists feel that germline editing could have a legitimate therapeutic use in the future in protecting against disease, or a valid research use, but they agree that safety concerns remain paramount (http:// www.nature.com/news/ethics-of-embryo-editing-divides-scientists1.17131).

Use of germline editing technologies for non-therapeutic (i.e., nondisease-related) modification of embryos is clearly unacceptable. Nor is it certain that attempts at modulating multigenic traits, such as eye color, would ever have the expected outcome, and there is no way to accurately extrapolate from animal experiments. But there may be instances of rare recessive diseases affecting both parents-such as sickle cell anemia or certain types of blindness-for which correction by gene editing would be the only way to ensure a healthy child. And the debate must consider such possibilities.

There are crucial safety concerns that would need to be resolved before clinical application could ever be considered. Some of these concerns include unintended mutations at the target DNA or at off-target sites, targeting of only one allele when biallelic targeting is necessary to prevent disease, and genetic mosaicism if all cells in the embryo are not targeted. However, it is feasible that technological improvements in targeting and screening of genetically modified embryos could eventually allay some of these concerns.

Discussions must therefore directly address considerations that would become realities should gene editing be approved for correction of disease genes in any country. For example, how would abuse of this technology to select phenotypes in an otherwise healthy embryo be regulated, how would children born using these gene modifying techniques-and their future offspring - be monitored, and how would society cope with potential disparities of access to and outcomes of the procedure?

There are also research precedents that may help inform these discussions. The UK recently approved mitochondrial replacement therapy in which an embryo is created that contains nuclear DNA from two parents and mitochondrial DNA from a third individual. And human somatic cell nuclear transfer (SCNT) into an enucleated oocyte has been recently successfully achieved in vitro. Both techniques involve germline changes to the human genome, and researchers involved in these types of studies could help inform the debate.

In 1975, Nobel laureate Paul Berg convened a conference at Asilomar in California to discuss potential biohazards of the use of recombinant DNA in response to fears resulting from his work with the polyomavirus SV40 and which had led to a brief pause of experiments using recombinant DNA. Concerns at the time included the potential for release of a recombinant organism into the environment with unanticipated effects on human health or on animal or plant ecosystems. The meeting participants agreed that research using the new technology should be allowed to proceed, but they also delineated guidelines to ensure its safe use and determined that stricter policies should be put in place at the outset and modified as more biological insight was gained (http:// authors.library.caltech.edu/11971/1/BERpnas75.pdf).

Similarly, in response to concerns about the use of human embryonic stem cells (hESCs), the International Society for Stem Cell Research (ISSCR) assembled a task force of experts from 14 countries to draft guidelines, published in 2007, on the use of hESCs, asking journal editors and granting agencies to ensure compliance in order to help translate the guidelines into practice. The guidelines prohibit the in vitro culture of human embryos used in research beyond 14 days as well as reproductive cloning, guidelines that should remain relevant in the current discussions. The ISSCR has now weighed in on germline editing, as has a group of scientists, lawyers and ethicists in a Perspective in Science, both calling for a halt on the clinical application of germline nuclear genome editing and recommending that in vitro genome editing research be allowed to proceed.

With rapid advances in genetic engineering technologies, it is now time to extend the discussion more globally to experts, legislators and patient advocates to analyze the risks and potential benefits of germline alterations, to draft guidelines for the safe and ethical use of germline editing technologies in research and potential clinical settings, and to recognize that with greater knowledge will come more meaningful regulation. 